Article

\title{
Development and Characterization of a Poly (Vinyl Alcohol)/Graphene Oxide Composite Hydrogel as An Artificial Cartilage Material
}

\author{
Yibo Zhao ${ }^{1}$, Wataru Terai ${ }^{1}$, Yuko Hoshijima ${ }^{2}$, Kazuma Gotoh ${ }^{2}$, Koji Matsuura ${ }^{2,3}$ (D) \\ and Kazuaki Matsumura ${ }^{1, *(\mathbb{D})}$ \\ 1 School of Materials Science, Japan Advanced Institute of Science and Technology, 1-1 Asahidai, Nomi, \\ Ishikawa 923-1292, Japan; s1820022@jaist.ac.jp (Y.Z.); s1430055@jaist.ac.jp (W.T.) \\ 2 Graduate School of Natural Science and Technology, Okayama University, 3-1-1 Tsushima-Naka Kita-Ku, \\ Okayama 700-8530, Japan; pgrs17ln@okayama-u.ac.jp (Y.H.); kgotoh@okayama-u.ac.jp (K.G.); \\ kojimatu@md.okayama-u.ac.jp (K.M.) \\ 3 Department of Biomedical Engineering, Faculty of Engineering, Okayama University of Science, \\ 1-1 Ridai-Cho Kita-Ku, Okayama 700-0005, Japan \\ * Correspondence: mkazuaki@jaist.ac.jp; Tel.: +81-761-51-1680
}

Received: 21 September 2018; Accepted: 13 November 2018; Published: 16 November 2018

\begin{abstract}
Poly (vinyl alcohol) hydrogel (PVA-H) is expected to be a suitable artificial articular cartilage material because of its high biocompatibility. However, it is difficult to affix to the surface of a living joint because it is bioinert and its mechanical strength needs to be improved. In this study, graphene oxide $(\mathrm{GO})$ subjected to two oxidation rounds was used to form a nanocomposite material and the composite hydrogel PVA-GO-H was prepared by low-temperature crystallization. Scanning electron microscope (SEM) images showed that the addition of GO can increase roughness of the hydrogel surface. Contact angle measurements showed that the surface of PVA-GO-H exhibited hydrophobicity that increased with GO concentration and not with that of PVA-H, indicating that the hydrophilic parts of PVA and GO form hydrogen bonds and the hydrophobic part of GO was exposed on the surface. Tensile tests demonstrated that Young's modulus was enhanced on the addition of GO. Osteoblast cells showed more affinity for PVA-GO-H than PVA-H, which much more cells adhere to than to PVA-GO-H after a certain period of culturing, suggesting GO can improve the cell attachment of PVA-H. Further studies on the influence of the oxidation time of GO are still required.
\end{abstract}

Keywords: PVA; graphene oxide; composite hydrogel; biomaterial

\section{Introduction}

The hip joint consists of an articulation between the head of the femur and acetabulum of the pelvis, which supports most of the load on joints and can bear up to approximately 5-7 times the body weight in some cases [1]. A cartilage layer 2 to $4 \mathrm{~mm}$ thick covering the femoral head, called the articular cartilage, plays an important role in the function of the joint, in that it absorbs the impact applied to the hip joint and prevents friction between bones. Cartilage tissue has low possibilities of self-repair because it does not receive an adequate supply of the requisite nutrients essential for such repair. Once damaged, a replacement surgery must therefore be carried out. Currently, however, it is estimated that approximately $10 \%$ of people over the age of 60 years suffer from clinical symptoms caused by osteoarthritis [2], a type of joint condition that results from the breakdown of articular cartilage. For such articular cartilage damage, a newly developed technique-specifically, resurfacing hip arthroplasty (RHA) [3] — is considered a highly reliable treatment to recover the normal function 
of the hip joint, in which only the affected cartilage needs to be replaced with artificial cartilage. However, artificial cartilage materials for resurfacing in RHA have very demanding requirements: they need to have similar mechanical properties as that of human cartilage and exhibit features such as low wear, nontoxicity, and biocompatibility.

Hydrogel, compared to normal cartilage materials such as metals and ceramics, is considered to have better biocompatibility owing to its ability to hold water [4]. Furthermore, recent studies have shown that the frictional coefficient of hydrogel decreases in the presence of branched polymer chains [5], demonstrating its suitability for use as an artificial articular cartilage material. Physically crosslinked polyvinyl alcohol hydrogel (PVA-H) is considered to be a good artificial cartilage material owing to its similar friction behavior as that of cartilage and its porous structure, which closely resembles cartilage. However, PVA-H is known to be a bioinert material and is therefore extremely difficult to adhere and affix to the surface of a living joint [6]. Extensive research has been undertaken in improving the mechanical strength and cell compatibility of hydrogels [7-12]. For instance, some have suggested preparing double-network (DN) hydrogels, and found that PAMPS/PAAm DN gels sustained more than 20 times the stress that PAMPS/PAAm SN (single-network) gels can withstand [8]. Some researchers blended PVA with chitosan and gelatin and achieved better protein adsorption and higher cell proliferation on PVA composite gels $[9,10]$. Others, on the other hand, have combined $\mathrm{Al}_{2} \mathrm{O}_{3}$ or Ti with PVA-H and succeeded in implanting the composite material in a dog' femur [11].

Graphene oxide (GO) is single-layer graphene with oxygen functionalities and is known as an excellent nanofiller. GO not only exhibits the physical properties of graphene but also shows dispersibility in water and other organic solvents because of the presence of oxygen functionalities. This important property enables mixing of GO with PVA on a molecular level. Some studies [12-16] on PVA/GO composite materials reported good dispersion of GO in a PVA matrix. The addition of GO enhanced the mechanical strength [12-15] because GO integrated with PVA with strong hydrogen bonds and the external force could be transferred to GO, which has high mechanical strength. Furthermore, GO is reported to present no toxicity issue and can adhere to cells $[12,15,16]$ : therefore, it is expected to be an excellent biomaterial in bone tissue engineering, drug delivery, and other biological fields.

In this study, GO was used as a composite material to prepare a PVA/GO composite hydrogel (PVA-GO-H). To improve the fixation of the articular cartilage into the defect, good adhesion on the direct contact surface between PVA-H and underlying bone is necessary [11,17]. Therefore, we expected that GO composition could enhance the adhesion to the bone. Several methods are used to prepare PVA-H, such as the cast-drying method (CD) [18], the freeze-thawing method (FT) [19], and low-temperature crystallization (LTC) [20]. In general, CD is used to prepare hydrogels with high strength and transparency. However, it is unlikely to obtain PVA-GO-H with good dispersion by the $\mathrm{CD}$ method because of the precipitation of GO in solution. Consequently, we used the LTC method to prepare PVA-GO-H. PVA-H and PVA-GO-H with different GO concentrations prepared using different oxidation times for GO were prepared and then their contact angles, mechanical properties, and biocompatibilities were investigated. As a result of our work, high-water-content hydrogels suitable as artificial cartilage materials with excellent mechanical properties and biocompatibilities could be anticipated.

\section{Materials and Methods}

\subsection{Preparation of $G O$}

Graphite powder, sulfuric acid $\left(\mathrm{H}_{2} \mathrm{SO}_{4}\right)$, phosphorus oxide $\left(\mathrm{P}_{2} \mathrm{O}_{5}\right)$, potassium permanganate $\left(\mathrm{KMnO}_{4}\right)$, and hydrogen peroxide $\left(\mathrm{H}_{2} \mathrm{O}_{2}\right)$ were purchased from Wako Chemical Co., Ltd. (Osaka, Japan). Potassium persulfate $\left(\mathrm{K}_{2} \mathrm{~S}_{2} \mathrm{O}_{8}\right)$ and sodium nitrate $\left(\mathrm{NaNO}_{3}\right)$ were obtained from Nakarai Tesque Co., Ltd. (Kyoto, Japan). GO was prepared by the Hummers method [21,22]. First, $6 \mathrm{~g}$ of graphite powder, $50 \mathrm{~mL}$ of $\mathrm{H}_{2} \mathrm{SO}_{4}$, and $10 \mathrm{~g}$ of $\mathrm{K}_{2} \mathrm{~S}_{2} \mathrm{O}_{8}$ were mixed, and the temperature of 
the mixture was elevated to $80^{\circ} \mathrm{C} . \mathrm{P}_{4} \mathrm{O}_{10}$ was slowly added to the mixture, which was stirred for $3 \mathrm{~h}$. The mixture was filtered and the filtrated powders were dried overnight. Then, $150 \mathrm{~mL}$ of $\mathrm{H}_{2} \mathrm{SO}_{4}$ was added to the dried solid at $4{ }^{\circ} \mathrm{C}$, and $6.5 \mathrm{~g}$ of $\mathrm{NaNO}_{3}$ and $20 \mathrm{~g}$ of $\mathrm{KMnO}_{4}$ were slowly added to the suspension. Subsequently, the ice bath was removed and the temperature of the suspension maintained at $35{ }^{\circ} \mathrm{C}$ for 0.5 or $30 \mathrm{~h}$. After the oxidation process, $305 \mathrm{~mL}$ of distilled water (DW) was added, and the suspension was allowed to rest for $0.5 \mathrm{~h}$. It was then diluted with DW to a total volume of $620 \mathrm{~mL} .33 \mathrm{~mL}$ of $\mathrm{H}_{2} \mathrm{O}_{2}$ was added to remove residual $\mathrm{KMnO}_{4}$ and $\mathrm{MnO}_{2}$, and the resulting suspension was stirred for $0.5 \mathrm{~h}$. After dilution of the suspension with $500 \mathrm{~mL}$ of DW, the supernatant was removed. The $\mathrm{pH}$ of the GO suspension increased on DW dilution using a crossflow system with a hollow fiber (Spectrum, Repligen Co., Ltd., Waltham, MA, USA) and centrifugation (8000 rpm, $5 \mathrm{~min}$ ) several times. We denoted GO samples subjected to the oxidation process for 0.5 and $30 \mathrm{~h}$ by $\mathrm{KMnO}_{4}$ as $\mathrm{GO}(0.5 \mathrm{~h})$ and $\mathrm{GO}(30 \mathrm{~h})$, respectively.

\subsection{X-ray Photoelectron Spectroscopy (XPS) Spectrum Measurement of GO}

The elemental ratio of GO $(0.5 \mathrm{~h})$ and GO $(30 \mathrm{~h})$ was determined using X-ray photoelectron spectrometry (AXIS-ULTRA DLD, Shimadzu Crates Co., Ltd., Kyoto, Japan). The area ratios of each peak were obtained by XPSPEAK software and peak fitting was performed using a linear combination function of the Gaussian and Lorentz functions.

\subsection{Preparation of PVA-H/PVA-GO-H}

First, $90 \mathrm{~g}$ of a dimethyl sulfoxide (DMSO) $/ \mathrm{H}_{2} \mathrm{O}$ solution $\left(\mathrm{DMSO} / \mathrm{H}_{2} \mathrm{O}=80: 20 \mathrm{w} / \mathrm{w}\right.$ ) was prepared and GO $(0.5 \mathrm{~h})$ or GO $(3 \mathrm{~h})$ was dispersed in solution to obtain GO/DMSO/ $\mathrm{H}_{2} \mathrm{O}$ dispersions of different GO concentrations. Then, $10 \mathrm{~g}$ of PVA powder (degree of polymerization: 1700, degree of sapponification: 99.0\%; VF-17, JAPAN VAM \& POVAL Co., Ltd., Osaka, Japan) was dissolved in the dispersion and the mixture was stirred at $95^{\circ} \mathrm{C}$. Next, the dispersion was sealed in autoclave and maintained at $120^{\circ} \mathrm{C}$ for $30 \mathrm{~min}$ and then naturally cooled to room temperature to obtain a well-dispersed solution. The solution was then poured between two brass plates with a 3-mm-thick spacer and cooled to $-20^{\circ} \mathrm{C}$ for $24 \mathrm{~h}$. After gelation, the obtained gels were immersed in ethanol at room temperature for three days, wherein the ethanol was exchanged twice a day to remove the DMSO. Then, the obtained hydrogels without DMSO were kept at room temperature for one day and subsequently dried under vacuum for three days to remove water and organic solvents remaining in the gels. An annealing treatment under vacuum was carried out at different temperatures to control the water content of the gels. Finally, dried gels were immersed in distilled water for three days to rehydrate. The water content (WC) of the hydrogels was calculated using Equation (1)

$$
\mathrm{WC}=\frac{W_{\text {wet }}-W_{\text {dry }}}{W_{\text {wet }}} \times 100 \%,
$$

where $W_{\text {wet }}$ is the weight of the rehydrated gel and $W_{d r y}$ is the weight of the dried gel after annealing treatment.

\subsection{SEM Measurements of PVA-H/PVA-GO-H}

In order to obtain information about the surface topography of the gels, a scanning electron microscope (SEM) (S-5200, Hitachi High-Technologies Co., Ltd., Tokyo, Japan) was used to obtain SEM images of PVA-H and PVA-GO-H at $0.05 \%$ and $0.40 \%$ GO concentrations.

\subsection{Contact Angle Measurements}

Hydrophilicity was evaluated using an automatic contact angle meter (DM-301, Kyowa Interface Science Co., Ltd.) and image analysis software (FAMAS, Kyowa Interface Science Co., Ltd., Saitama, Japan). First, the obtained gels were placed on the DM-301. Then, a $2 \mu \mathrm{L}$ droplet of water 
was dropped onto the gel. The contact angle of the dropped droplets was measured using the contact angle meter.

\subsection{Tensile Test}

A universal testing machine (Autograph, Shimadzu Co., Ltd., Kyoto, Japan) was used to determine the tensile mechanical properties of the hydrogels. The samples were cut to JIS dumbbell 7 type specimens (gauge length: $25 \mathrm{~mm}$, width: $2 \mathrm{~mm}$ ). To eliminate the effect of deflection of the gels, an initial load of $0.01 \mathrm{~N}$ was applied. The test was performed at a speed of $5 \mathrm{~mm} / \mathrm{min}$ and was repeated at least three times to ensure reproducibility.

\subsection{Cell Culture}

The hydrogels were expected to be artificial cartilage materials; therefore, in order to confirm the adhesion of gel to bone, osteoblast cells MC3T3, which were arranged on the surface of bone tissue, were seeded on gels to evaluate cell attachment and proliferation. The gels were cut into circles with a diameter of $10 \mathrm{~mm}$ and immersed in $70 \%$ ethanol for two days to sterilize, followed by immersion in Dulbecco's modified Eagle medium (DMEM) to rehydrate. The rehydrated gels were placed in the well of a culture plate (24-well multiplate, Iwaki, Japan). Then, $1 \mathrm{~mL}$ of a suspension of osteoblast $\left(2 \times 10^{4}\right.$ cells per $\left.\mathrm{mL}\right)$ in DMEM containing $10 \%$ fetal bovine serum (FBS) was added to the well. The cells were then cultured at $37^{\circ} \mathrm{C}$ in $5 \% \mathrm{CO}_{2}$ for a given period. After culturing, the cells were fluorescent stained by Calcein-AM (Dojindo, Kumamoto, Japan), and the fluorescence of the cells attached to the gels was observed with a fluorescence microscope (Biozero, Keyence, Osaka, Japan). To infer the number of surviving cells on gels objectively, the gels were immersed in trypsin at $37^{\circ} \mathrm{C}$ to separate the cells from the gels, and the number of living cells were counted via trypan-blue staining. In addition, to evaluate the initial cell adhesiveness of the gels, a $10 \mu \mathrm{L}$ concentrate of $10 \%$ FBS-DMEM containing $2 \times 10^{4}$ osteoblast cells were added to the surface of the gels and cultured at $37^{\circ} \mathrm{C}$ in $5 \%$ $\mathrm{CO}_{2}$ for $2 \mathrm{~h}$, followed by observation of the fluorescence and cell counting.

\section{Results}

\subsection{XPS Spectrum of GOs}

The obtained elemental ratio of GO is shown in Table 1. The ratio of $\mathrm{C}$ and $\mathrm{O}$ was roughly $\mathrm{C}: \mathrm{O}=7: 3$, suggesting $\mathrm{GO}$ was successfully obtained, and a slight change in the ratio was observed with a change in the oxidation time.

Table 1. Atomic ratio of carbon and oxygen in GO (graphene oxide) as determined by XPS.

\begin{tabular}{ccc}
\hline Oxidation Time (h) & $\mathbf{0 . 5}$ & $\mathbf{3 0}$ \\
\hline $\mathrm{C}: \mathrm{O}$ & $71: 28$ & $69: 29$ \\
\hline
\end{tabular}

For quantification of the degree of oxidation, C1s peak fitting profiles are shown in Figure 1 and the ratio of five peak-fitting areas to the area of the $\mathrm{C}-\mathrm{C}$ peak is shown in Table 2. From this result, no obvious change was seen in the ratio of functional groups owing to the different oxidation times of GO except hydroxyl $(\mathrm{OH})$. GO oxidized for $0.5 \mathrm{~h}$ has twice the amount of hydroxyl groups that $30 \mathrm{~h}$ oxidized GO has. This may be due to the hydroxyl groups being oxidized to carboxyl groups $(\mathrm{O}-\mathrm{C}=\mathrm{O})$ or carbonyl groups $(\mathrm{C}=\mathrm{O})$ with increasing oxidation time. The proportions of both carbonyl groups and carboxyl groups slightly increased with the oxidation time, as shown in Table 2. GO ( $0.5 \mathrm{~h})$ contained more hydroxyl groups and is thus likely to have better mechanical properties as it is expected to exhibit stronger interactions with PVA owing to the hydrogen bonds. 
a)

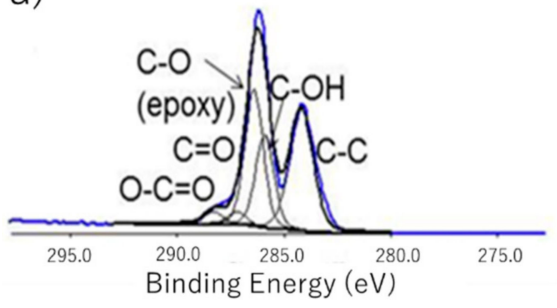

b)

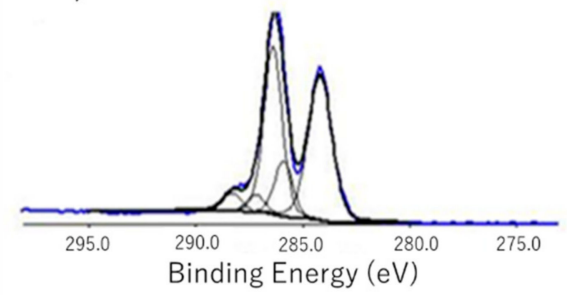

Figure 1. C1s peak-fitting profile of (a) GO (0.5 h) and (b) GO (30 h).

Table 2. Ratio of various functional groups to C-C peak of GO as determined by XPS.

\begin{tabular}{ccc}
\hline Functional Group & GO (0.5 h) & GO (30 h) \\
\hline C-C & 1.00 & 1.00 \\
C-OH & 0.60 & 0.30 \\
C-O (epoxy) & 0.87 & 0.90 \\
C=O & 0.087 & 0.094 \\
O-C $=\mathrm{O}$ & 0.082 & 0.099 \\
\hline
\end{tabular}

\subsection{SEM Images of $P V A-H / P V A-G O-H$}

SEM images of PVA-H and PVA-GO-H of $0.05 \%$ and $0.4 \%$ GO concentration are shown in Figure 2. As shown in Figure 2, no visible pore structures were seen in the top layers of the gels. In addition, the surface of the gels of high GO concentration (Figure 2d,e) were observed to be rugged, indicating that the addition of GO increased the roughness of the gel surfaces. GO flakes were distributed disorderly on the surface and some parts formed a few crosslinking points, whereas some parts formed more, thereby causing these rough structures on the surface.

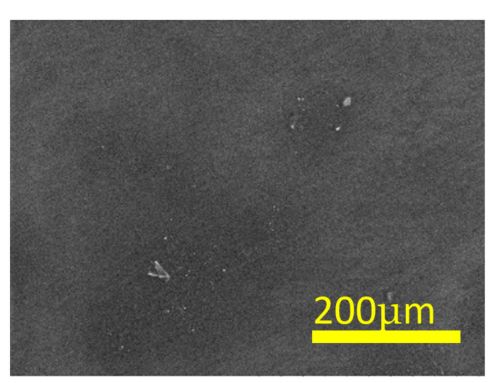

(a)

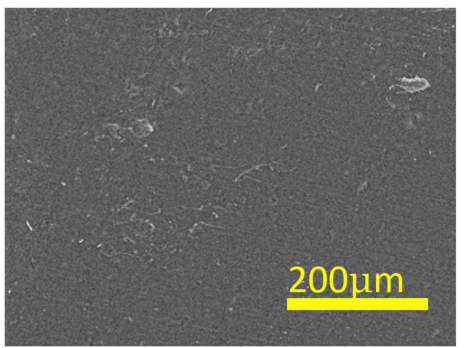

(b)

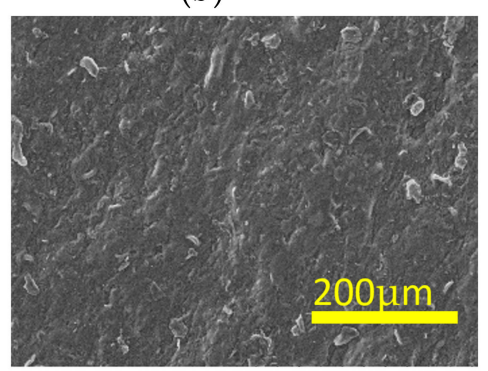

(d)

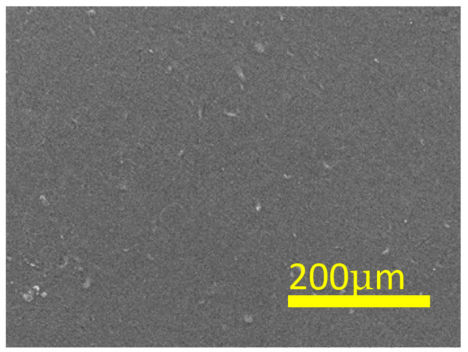

(c)

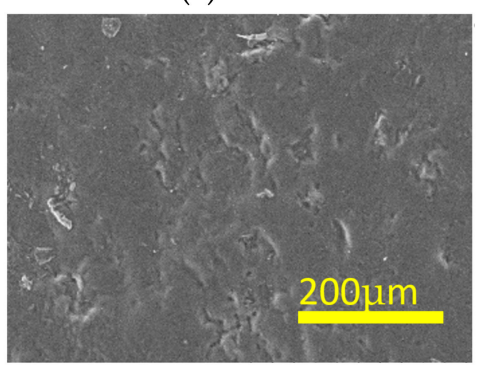

(e)

Figure 2. SEM images of (a) PVA-H and PVA-GO-H. Oxidation time and concentration of GO: (b) $0.5 \mathrm{~h}$ $0.05 \%$, (c) 30 h $0.05 \%$, (d) 0.5 h $0.4 \%$, (e) 30 h $0.4 \%$.

\subsection{Hydrophilicity Evaluation}

The results of contact angle tests of the hydrogels are shown in Figure 3. It was found that the surface of PVA-GO-H exhibited hydrophobicity with increasing GO concentration compared to that of PVA-H. It appeared that the hydrophilic part of PVA and GO formed hydrogen bonds 
and the hydrophobic part of GO was exposed on the surface. Moreover, no significant difference was observed for the different oxidation times of GO. It is known that differences of surface properties including hydrophilicity affect cell attachment and proliferation, leading to different behaviors of seeded cells on gels.

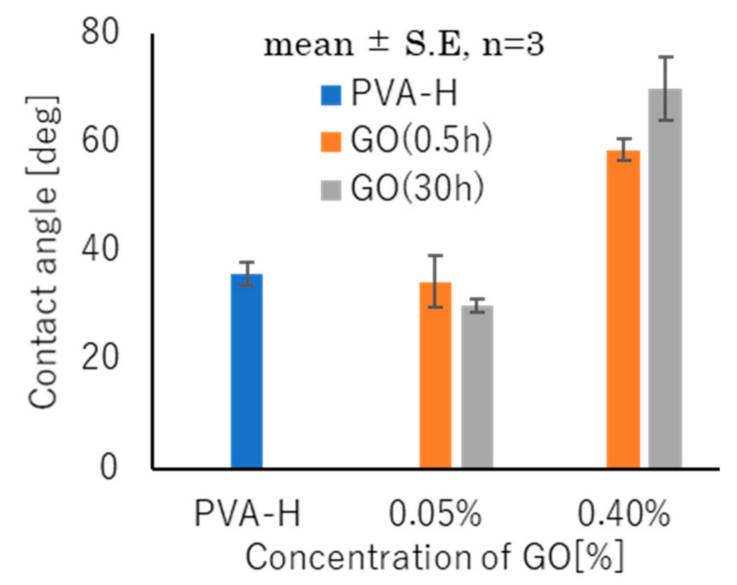

Figure 3. Contact angle of PVA-GO-H. (S.E.: standard error)

\subsection{Control of Water Content}

The water contents of each sample under a variety of heating conditions are shown in Figure 4, where samples subjected to $160^{\circ} \mathrm{C}$ for $4 \mathrm{~h}$ and $180{ }^{\circ} \mathrm{C}$ for $2 \mathrm{~h}$ are denoted as $160-4$ and $180-2$, respectively. The gels subjected to treatment at $160^{\circ} \mathrm{C}$ showed a much lower water content compared to the untreated samples. The gels under the $180^{\circ} \mathrm{C}$ treatment showed an even lower water content. The crystallization degrees of gels can be increased by annealing treatment under vacuum [23]. Gels with high degrees of crystallization are believed to have access to less water and thus exhibit low water content. In addition, the WC (water content) values of the gels subjected to annealing treatment were larger than that of the untreated gels. This can be explained by the surface of the cut gel not being flat. The uneven surface was caused during the drying process of the gels; thus, it is necessary to fix the gels to avert curling while drying.

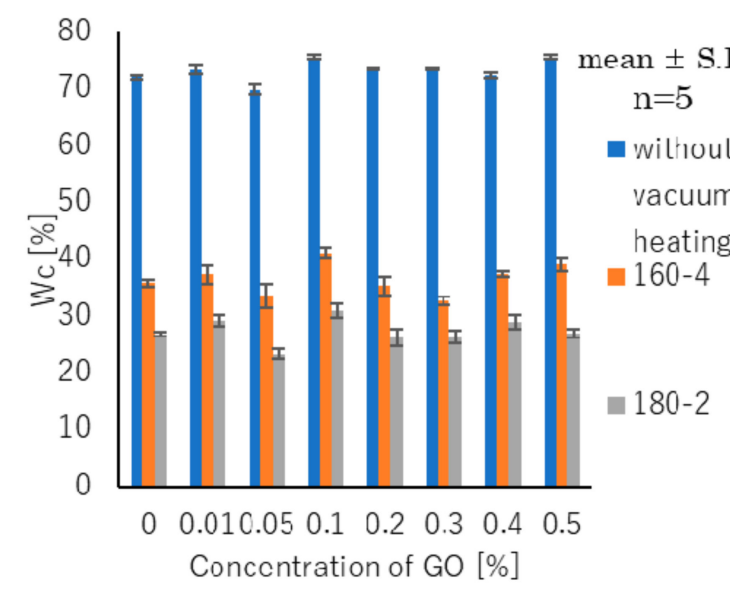

(a)

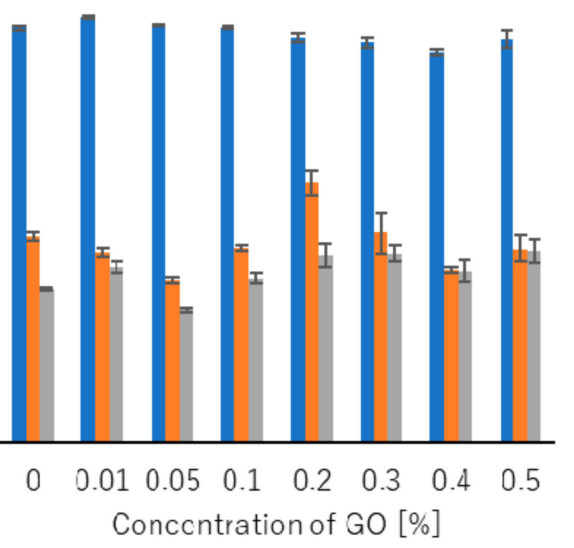

(b)

Figure 4. Contact angle of PVA-GO-H with (a) GO (0.5 h) and (b) GO (30 h).

It is worth mentioning that the untreated gels exhibited a water content of around $70 \%$, which is close to that of human cartilage. Because the crystallization degree has a significant influence on the mechanical properties, the mechanical strength of gels can be controlled by 
different annealing temperatures and it is expected that we can prepare gels with high strength by such annealing treatments.

\subsection{Mechanical Properties}

Hydrogels with different water content (WC) values were obtained, as mentioned in Section 3.3. It is necessary to evaluate the Young's modulus of gels at an identical WC. However, even if a hydrogel is produced under the same condition, an error may occur in determining the value of the WC, which affects the measurement result.

In order to eliminate the influence of $\mathrm{WC}$, a tensile test was carried out by using samples immediately after gelation (before immersion in ethanol). The result is shown in Figure 5. PVA-GO-H was found to have a higher Young's modulus than PVA-H and a significant improvement in the Young's modulus was observed with increasing GO concentration. For comprehensive analysis of the mechanical strength of gels, the Young's modulus of gels at different WC were measured-the result is shown in Figure 6. To compare the Young's modulus trend of each sample, approximate curves are shown. Gels with low water content were observed to have high Young's modulus values and it was intuitively observed that the addition of GO can improve the Young's modulus of gels. However, according to Figures 5 and 6, the oxidation time of GO appeared to have no significant influence on the measured Young's modulus.

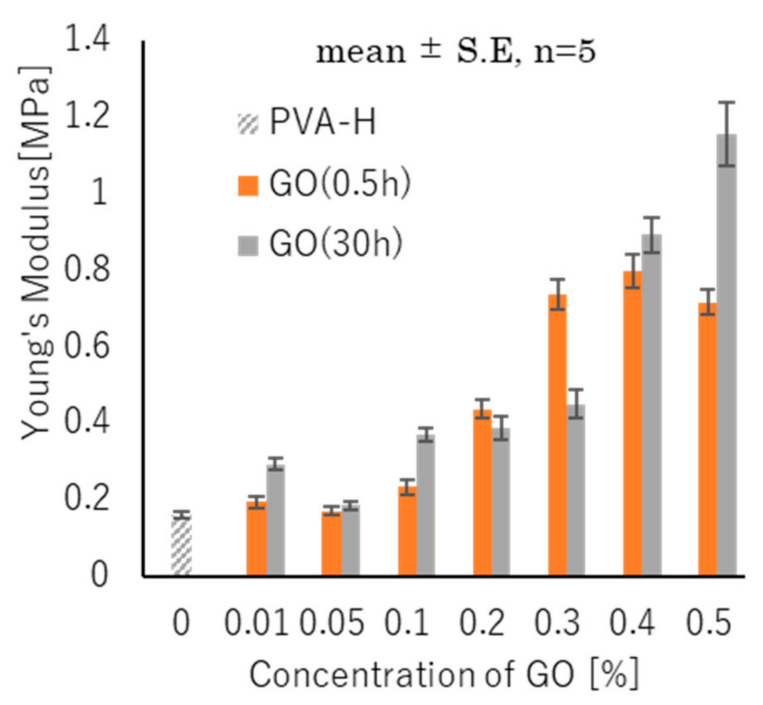

Figure 5. Young's modulus of PVA-GO-H.

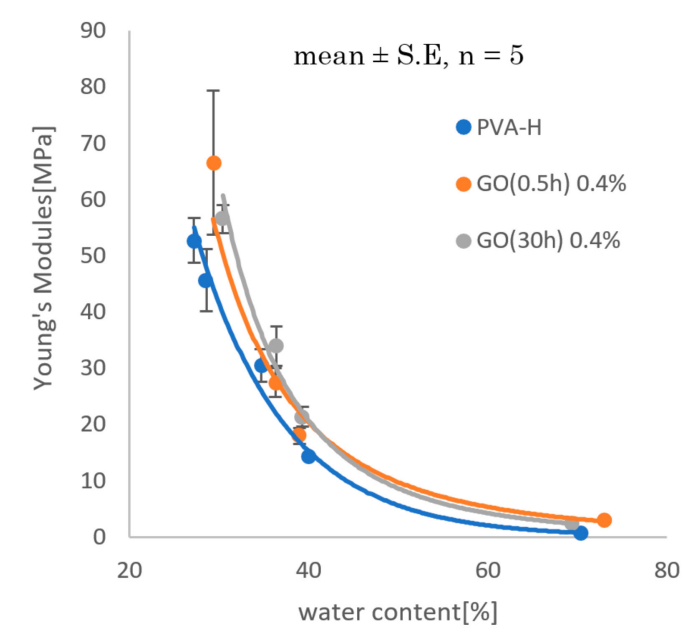

Figure 6. Young's modulus of PVA-GO-H at different WC (water content) values. 
Figure 7 shows the measured Young's modulus at a high WC. The difference in the Young's modulus between PVA-H and PVA-GO-H was clear even at high WC values where it exceeded 70\%. From this result, it can be suggested that hydrogels with both high moisture content and high strength can be produced by adding GO.

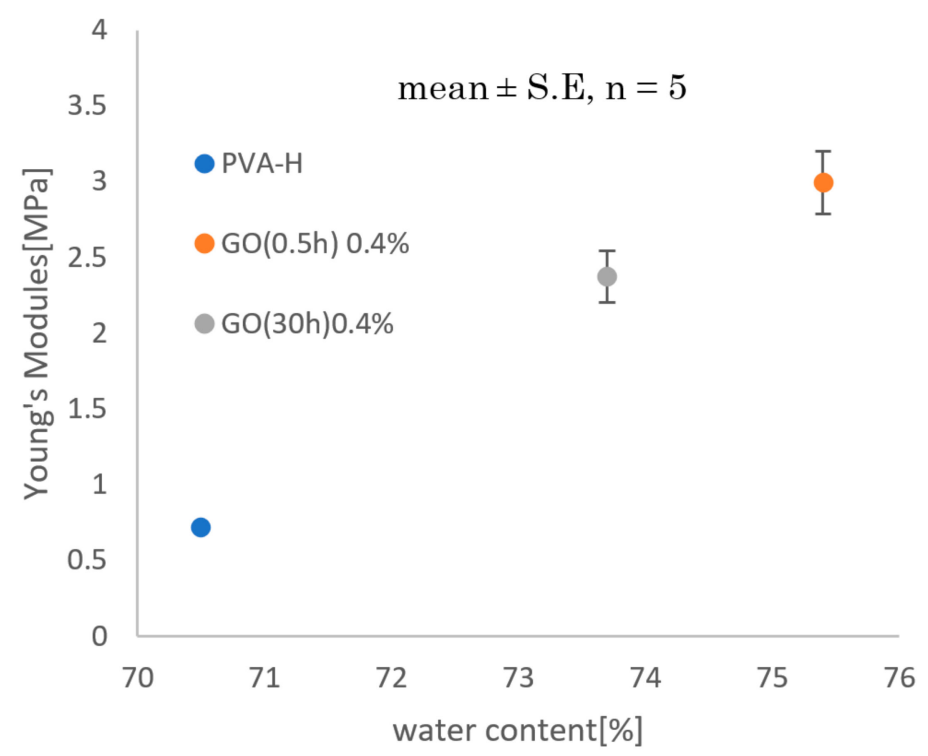

Figure 7. Comparison of Young's modulus of different samples at high WC (water content).

\subsection{Cell Attachment and Proliferation}

The results of fluorescence observation and cell counting are shown in Figures 8-13. The initial cell attachment can be evaluated by the results of fluorescence observation and cell counting of $2 \mathrm{~h}$ cultured cells. As mentioned in Section 2.7, a $10 \mu \mathrm{L}$ concentrate of osteoblast cells was injected onto the surface of the gel so as not to have it flow down the gel, excluding external factors such as precipitation or uneven dispersion of cells. As shown in Figure 8, many more cells adhered to PVA-GO-H than to PVA-H after $2 \mathrm{~h}$ of inoculation. The gels were washed twice by phosphate buffer solution PBS before counting so the numbers of cells shown in Figure 9 are believed to reflect the cell adhesion state. The numbers of adhered cells increased with increasing GO concentration, revealing that GO can improve cell attachment and that the GO surface is a good environment for cell proliferation.

Similar results appeared after three days' culturing, as shown in Figures 10 and 11. The variation in the number of attached cells between samples prepared under the same conditions was very large, which may be due to the difference in surface texture and incomplete detachment of cells when counting the number of adherent cells. It was confirmed that GO can improve cell attachment in the initial culture, whereas the oxidation time of GO appeared to have no significant influence on cell compatibility, based on the results of cell count (Figures 9 and 11). 


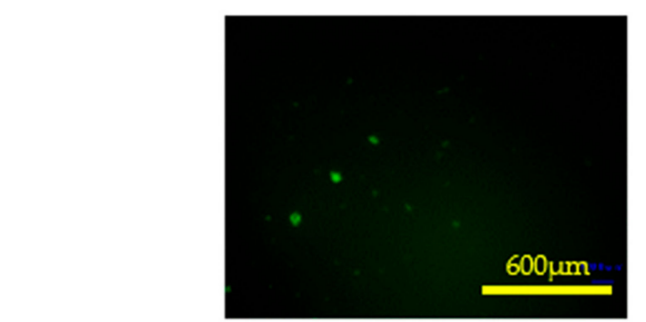

(a)

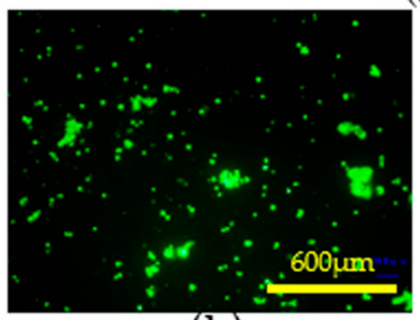

(b)

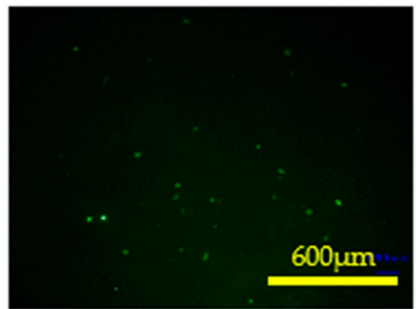

(d)

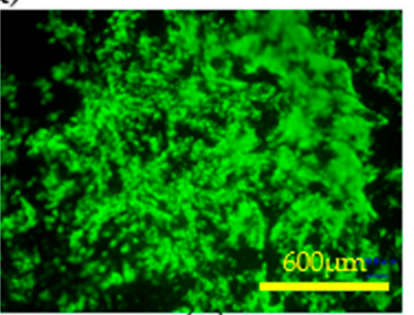

(c)

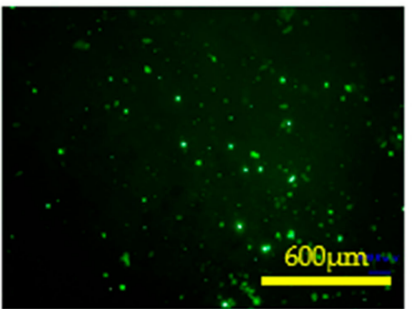

(e)

Figure 8. Fluorescence of cells cultured for $2 \mathrm{~h}$ : (a) PVA-H, (b) $0.05 \% \mathrm{GO}(0.5 \mathrm{~h})$, (c) $0.40 \% \mathrm{GO}(0.5 \mathrm{~h})$, (d) $0.05 \%$ GO (30 h), and (e) $0.40 \%$ GO (30 h).

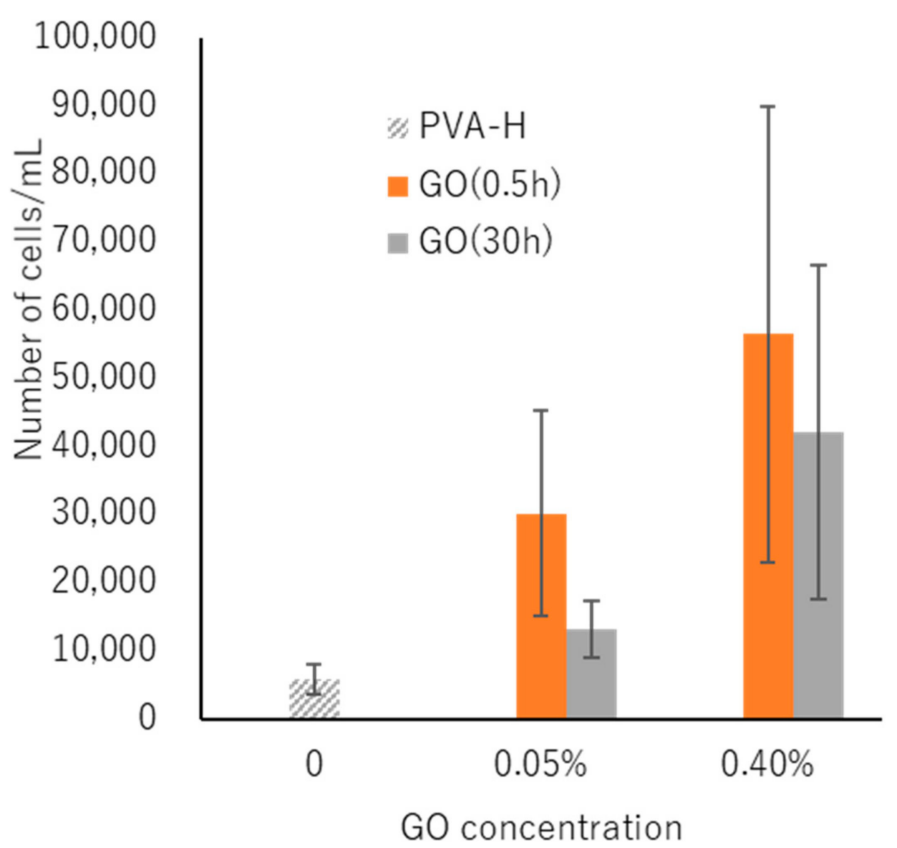

Figure 9. Number of cells attached to gels after $2 \mathrm{~h}$ of culturing. 


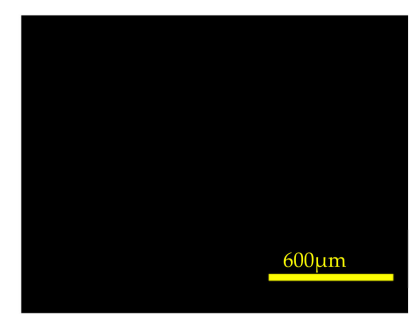

(a)

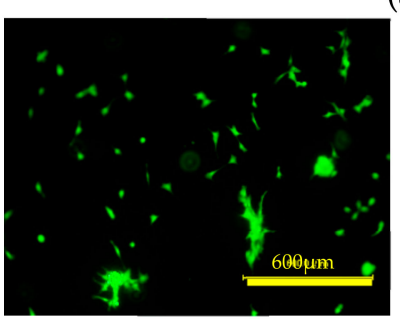

(b)

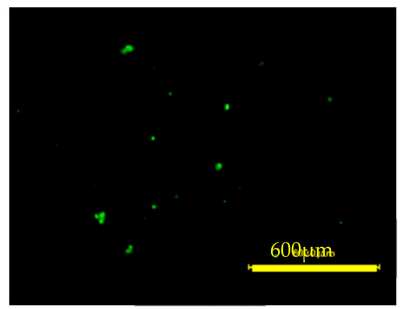

(d)

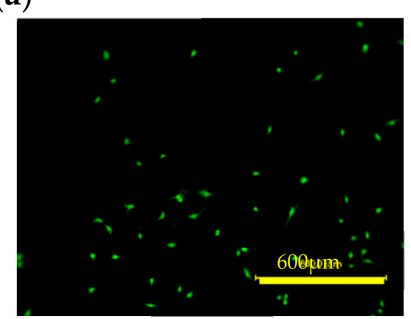

(c)

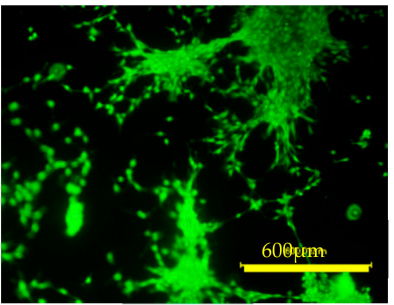

(e)

Figure 10. Fluorescence cells cultured for three days: (a) PVA-H, (b) $0.05 \%$ GO (0.5 h), (c) $0.40 \%$ GO $(0.5 \mathrm{~h}),(\mathbf{d}) 0.05 \% \mathrm{GO}(30 \mathrm{~h})$, and (e) $0.40 \% \mathrm{GO}(30 \mathrm{~h})$.

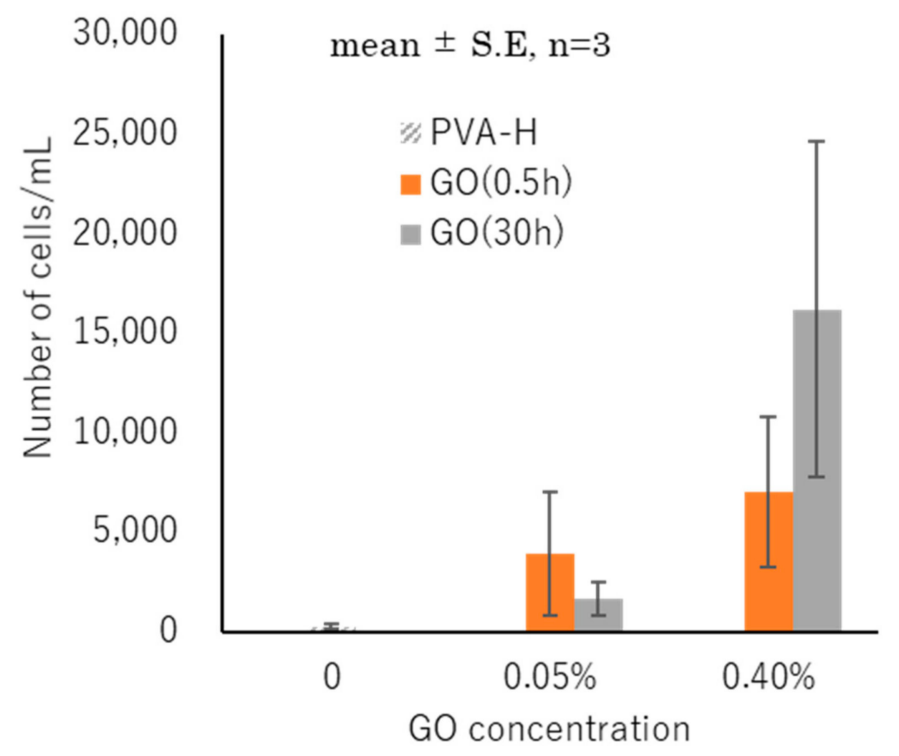

Figure 11. Number of cells attached to gels after three days of culturing.

Figures 12 and 13 show fluorescence observation and cell counting of a prolonged culture (12 days). It should be noted that these two groups of experiments (3 days and 12 days) did not use the same samples because the gels had to be abandoned after Calcein staining. The difference between the numbers of cells for 3 days and 12 days of culturing may be due to the variations of samples and experimental operations. Although the PVA-GO-H surfaces showed better cell adherence, the cells did not show an exponential growth in prolonged culturing except for the $0.4 \% 0.5$ h sample. However, the numbers of cells adhered on PVA-GO-H were still more than that of PVA-H, 
indicating that the GO surface is a good environment for cell proliferation. The $0.4 \% 0.5 \mathrm{~h}$ PVA-GO-H may be an excellent material, but further research is required to elucidate the mechanism.

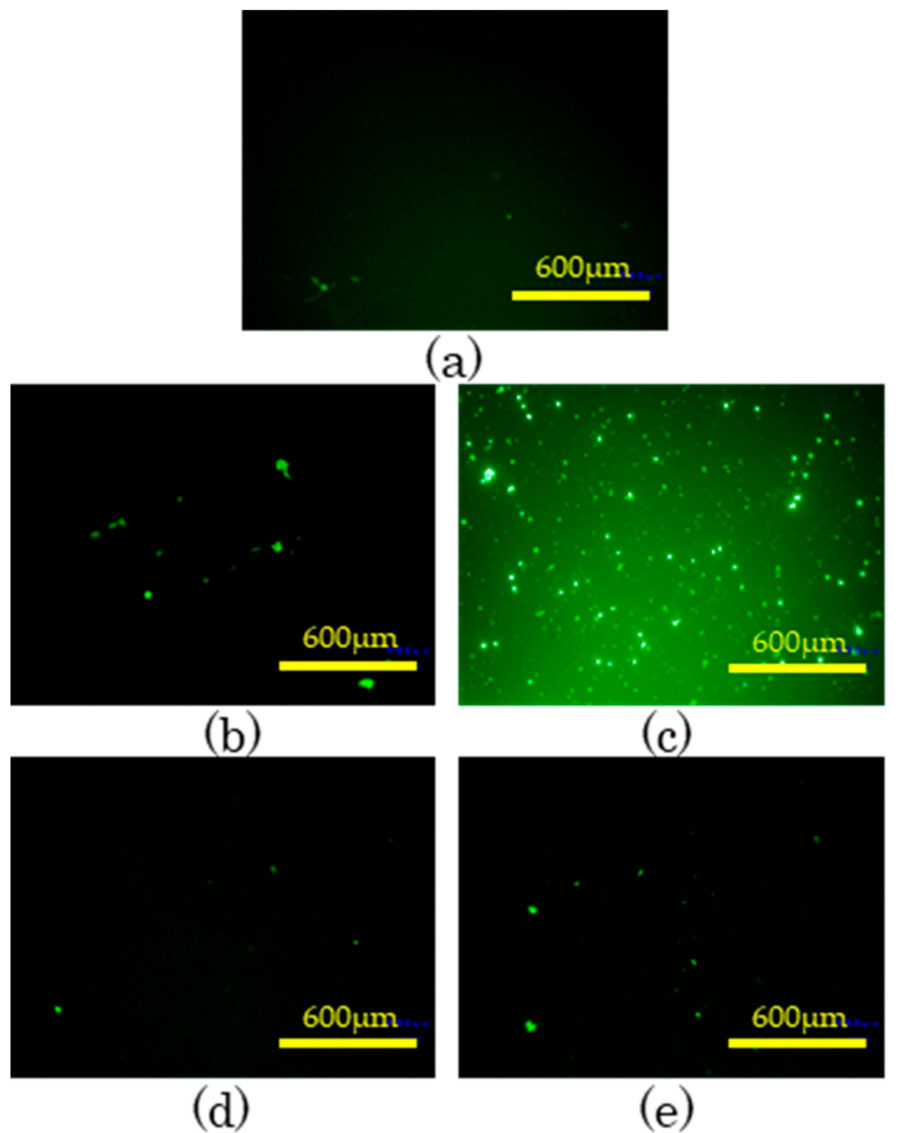

Figure 12. Fluorescence of cells cultured for 12 days: (a) PVA-H, (b) $0.05 \% \mathrm{GO}(0.5 \mathrm{~h})$, (c) $0.40 \% \mathrm{GO}$ $(0.5 \mathrm{~h}),(\mathbf{d}) 0.05 \% \mathrm{GO}(30 \mathrm{~h})$, and (e) $0.40 \% \mathrm{GO}(30 \mathrm{~h})$.

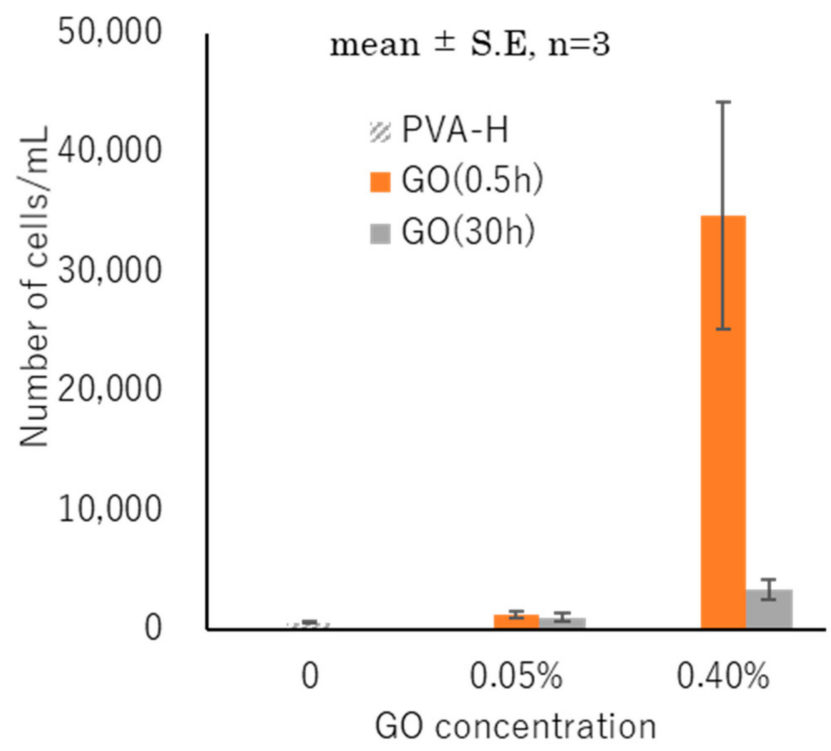

Figure 13. Number of cells attached to gels after 12 days culturing.

\section{Discussions}

Physically crosslinked PVA-GO-H was successfully prepared by low-temperature crystallization. The interaction between PVA and GO can be described as illustrated in Figure 14a, wherein the hydrogen 
atoms of the hydroxyl and carboxyl groups of GO interact with the oxygen atoms of the hydroxyl groups in PVA while the hydrogen atoms of the hydroxyl groups in PVA interact with the oxygen atoms of the hydroxyl, carboxyl, epoxy, and carbonyl groups in GO [15]. When mechanical load is applied to such a structure, GO is oriented in the tensile direction, as shown in Figure 14b. Consequently, the Young's modulus increases when GO is added. The effect of vacuum heating on the water content can be explained by the increasing density of microcrystals restricting the swelling ability [23-25].

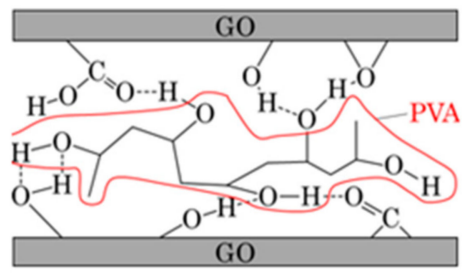

(a)

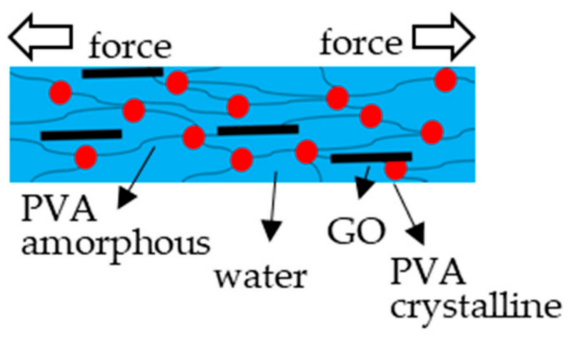

(b)

Figure 14. Schematic illustration of the mechanism of GO and PVA interactions. (a) Interactions between functional groups of PVA-H and GO. (b) Deformation model of PVA-GO-H during application of force.

From the tensile tests, GO was found to have a reinforcing effect on the Young's modulus. Unexpectedly, however, it was difficult to prepare PVA-GO-H with a GO concentration of over $0.4 \%$ owing to its high viscosity. The influence of the oxidation time of GO on the Young's modulus is still being estimated. By hydrophilicity evaluation, PVA-GO-H was found to exhibit hydrophobicity compared with PVA-H. With the addition of GO, the cell attachment of the gels improved obviously and it appeared that the rough structure observed in Section 3.2 is beneficial to cell adherence [16]. However, the cells did not proliferate as expected on PVA-GO-H after prolonged culturing. This can be explained as follows: osteoblasts cells are easy to adhere to where GO aggregates during initial culturing. However, because of the heterogeneous dispersion of GO, cells grow only in the areas where $\mathrm{GO}$ is exposed and are difficult to proliferate to the entire gel. Some residual ethanol on the hydrogel might cause a toxicity response that affects cell proliferation. Therefore, we need to develop a novel method of preparing PVA-H without using DMSO [24]. In conclusion, although we need to conduct further investigations into the mechanisms of biocompatibility of GO and detailed experiments for biomechanics, this nanocomposite approach might open a new path for high strength and highly biocompatible hydrogel materials with potential for artificial articular cartilages.

Author Contributions: Conceptualization, Writing-Review \& Editing, Supervision, Project Administration, K.M. (Kazuaki Matsumura); Methodology, Y.Z. and W.T.; Writing-Original Draft Preparation, Y.Z.; Validation, Formal Analysis; Y.Z.; W.T.; Y.H.; K.G.; and K.M.

Funding: This study was supported in part by the Center of Innovation Program "Construction of next-generation infrastructure using innovative materials: Realization of safe and secure society that can coexist with the Earth for centuries," from the Japan Science and Technology Agency.

Conflicts of Interest: The authors declare no conflict of interest.

\section{References}

1. Mayer, F.; Dickhuth, H.H. Physical activity after total joint replacement. Int. SportMed J. 2008, 9, 39-43.

2. Moti, L.T.; Hatem, E.S. Cartilage regeneration for treatment of osteoarthritis: A paradigm for nonsurgical intervention. Ther. Adv. Musculoskelet. Dis. 2015, 7, 76-87.

3. Wagner, H. Surface replacement arthroplasty of the hip. Clin. Orthop. Relat. Res. 1978, 134, 102-130. [CrossRef]

4. Allan, S.H. Hydrogels for biomedical applications. Adv. Drug Deliv. Rev. 2012, 64, 18-23.

5. Gong, J.P.; Kurokawa, T.; Narita, T.; Kagata, G.; Osada, Y.; Nishimura, G.; Kinjo, M. Synthesis of hydrogels with extremely low surface friction. J. Am. Chem. Soc. 2001, 123, 5582-5583. [CrossRef] [PubMed]

6. Yamaoka, T.; Tabata, Y.; Ikada, Y. Comparison of body distribution of poly(vinyl alcohol) with other water-soluble polymers after intra-venous administration. J. Pharm. Pharmacol. 1995, 47, 479-486. [CrossRef] [PubMed] 
7. Liu, Q.; Zheng, Y.; Wang, Y.; Wu, G. Review of poly(vinyl alcohol) hydrogel and its compounds in the application of artificial cartilage materials. J. Biomed. Eng. 2003, 20, 742-745.

8. Gong, J.P.; Katsuyama, Y.; Kurokawa, T.; Osada, Y. Double-network hydrogels with extremely high mechanical strength. Adv. Mater. 2003, 15, 1155-1158. [CrossRef]

9. Liu, Y.; Vrana, N.E.; Cahill, P.A.; McGuinness, G.B. Physically crosslinked composite hydrogels of PVA with natural macromolecules: Structure, mechanical properties, and endothelial cell compatibility. J. Biomed. Mater. Res. Part B 2009, 90B, 492-502. [CrossRef] [PubMed]

10. Koyano, T.; Minoura, N.; Nagura, M.; Kobayashi, K. Attachment and growth of cultured fibroblast cells on PVA/chitosan-blended hydrogels. J. Biomed. Mater. Res. 1998, 39, 486-490. [CrossRef]

11. Ushio, K.; Oka, M.; Hyon, S.-H.; Hayami, T.; Yura, S.; Matsumura, K.; Toguchida, J.; Nakamura, T. Attachment of artificial cartilage to underlying bone. J. Biomed. Mater. Res. B. 2004, 68B, 59-68. [CrossRef] [PubMed]

12. Qi, Y.Y.; Tai, Z.X.; Sun, D.F.; Chen, J.T.; Ma, H.B.; Yan, X.B.; Liu, B.; Xue, Q.J. Fabrication and characterization of poly(vinyl alcohol)/graphene oxide nanofibrous biocomposite scaffolds. J. Appl. Polym. Sci. 2013, 127, 1885-1894. [CrossRef]

13. Liang, J.J.; Huang, Y.; Zhang, L.; Wang, Y.; Ma, Y.F.; Guo, T.Y.; Chen, Y.S. Molecular-level dispersion of graphene into poly(vinyl alcohol) and effective reinforcement of their nanocomposites. Adv. Funct. Mater. 2009, 19, 2297-2302. [CrossRef]

14. Sharma, S.K.; Prakash, J.; Rujari, P.K. Effects of the molecular level dispersion of graphene oxide on the free volume characteristics of poly(vinyl alcohol) and its impact on the thermal and mechanical properties of their nanocomposites. Phys. Chem. Chem. Phys. 2015, 17, 29201-29209. [CrossRef] [PubMed]

15. Zhang, L.; Wang, Z.P.; Xu, C.; Li, Y.; Gao, J.P.; Wang, W.; Liu, Y. High strength graphene oxide/polyvinyl alcohol composite hydrogels. J. Mater. Chem. 2011, 21, 10399-10406. [CrossRef]

16. Shi, X.T.; Chang, H.X.; Chen, S.; Lai, C.; Khademhosseini, A.; Wu, H.K. Regulating cellular behavior on few-layer reduced graphene oxide films with well-controlled reduction states. Adv. Funct. Mater. 2012, 22, 751-759. [CrossRef]

17. Matsumura, K.; Hayami, T.; Hyon, S.H.; Tsutsumi, S. Control of proliferation and differentiation of osteoblasts on apatite-coated poly(vinyl alcohol) hydrogel as an artificial articular cartilage material. J. Biomed. Mater. Res. Part A 2010, 92A, 1225-1232. [CrossRef] [PubMed]

18. Komatsu, M.; Inoue, T.; Miyasaka, K. Light-scattering studies on the sol-gel transition in aqueous solutions of poly(vinyl alcohol). Polym. Prepr. Jpn. 1986, 24, 303-311. [CrossRef]

19. Peppas, N.A. Turbidimetric studies of aqueous poly(vinyl alcohol) solutions. Makromol. Chem. 1975, 176, $3433-3440$. [CrossRef]

20. Hyon, S.-H.; Cha, W.-I.; Ikada, Y. Preparation of transparent poly(vinyl alcohol) hydrogel. Polym. Bull. 1989, 22, 119-122. [CrossRef]

21. William, S.H.; Richard, E.O. Preparation of graphitic oxide. J. Am. Chem. Soc. 1958, 80, 1339-1339.

22. Matsuura, K.; Umahara, Y.; Gotoh, K.; Hoshijima, Y.; Ishida, H. Surface modification effects on the tensile properties of functionalised graphene oxide epoxy films. RSC Adv. 2018, 8, 9677-9684. [CrossRef]

23. Cha, W.I.; Hyon, S.H.; Ikada, Y. Microstructure of poly(vinyl alcohol) hydrogels investigated with differential scanning calorimetry. Die Makromol. Chem. 1993, 194, 2433-2441. [CrossRef]

24. Sakaguchi, T.; Nagano, S.; Hara, M.; Hyon, S.H.; Patel, M.; Matsumura, K. Facile preparation of transparent poly (vinyl alcohol) hydrogels with uniform microcrystalline structure by hot-pressing without using organic solvents. Polym. J. 2017, 49, 535-542. [CrossRef]

25. Otsuka, E.; Komiya, S.; Sasaki, S.; Xing, J.W.; Bando, T.; Hirashima, Y.; Sugiyama, M.; Suzuki, A. Effects of preparation temperature on swelling and mechanical properties of PVA cast gels. Soft Matter 2012, 8, 812-8136. [CrossRef]

(C) 2018 by the authors. Licensee MDPI, Basel, Switzerland. This article is an open access article distributed under the terms and conditions of the Creative Commons Attribution (CC BY) license (http:/ / creativecommons.org/licenses/by/4.0/). 